\title{
A Model for the Traveling Salesman Problem including the EC Regulations on Driving Hours
}

\author{
Herbert Kopfer and Christoph Manuel Meyer \\ Chair of Logistics, University of Bremen, Wilhelm-Herbst-Strasse 5, 28359 \\ Bremen; kopfer@uni-bremen.de, cmmeyer@uni-bremen.de
}

\section{Introduction}

Since April 2007 the new EC Regulation No 561/2006 concerning driving hours in road transport is effective. This regulation restricts the length of time periods for driving and requires minimum breaks and rest periods for drivers [2]. An analysis of the EC Regulation with respect to vehicle routing can be found in [3]. In this paper the restrictions on driving times and the need for breaks are formalized and integrated in an optimization model of the TSPTW. The solution space of the extended traveling salesman problem with time windows and EUconstraints (TSPTW-EU) contains all Hamiltonian circuits which fulfil the given time windows and restrictions of the Regulation relevant for a time period up to one week. The presented approach for extending the TSPTW to the TSPTW-EU is also applicable for the extension of the VRPTW and PDPTW, thus offering a possibility to include the EC Regulations in vehicle routing and scheduling.

\section{Integration of the EC Regulation into the TSPTW}

For the integration of the Regulation a position-based formulation of the TSPTW is used since it allows the calculation of driving times of sub-routes. It represents the position $\mathrm{q}$, at which a location $\mathrm{j}$ will be visited within a route (see e.g. [1]). For its formulation the following sets and variables are introduced.

I: set of locations $i \in\{0,1, \ldots, n\}$ with 0 as starting point of the route $\mathrm{P}: I \backslash\{0\}$

$\mathrm{Q}(\mathrm{j})$ : set of positions $\mathrm{q}$, at which location $\mathrm{j}$ can occur

$I(j, q)$ : set of locations $i$, from which can be traveled to $j$, so that $j$ occurs 
at position $\mathrm{q}$

$\mathrm{J}(\mathrm{i})$ : set of locations $\mathrm{j}$, to which can directly be traveled from $\mathrm{i}$

$J(i, q)$ : set of locations $j$, to which can be traveled from $i$, so that $j$ occurs at position $\mathrm{q}$

$Q(i, j)$ : set of positions $q$, at which $j$ can occur, if $j$ is reached from $i$

We assume that each driver is assigned to a fixed vehicle. In this case the driving times of a driver can simply be modeled by the wheel turning times of the assigned vehicle. The goal of the TSPTW-EU consists in minimizing the total time used for the route including breaks. This results in the objective function (1) where $t_{\text {rueck }}$ denotes the time when the route is completed.

$$
\text { Minf }=t_{\text {rueck }}
$$

Let $w_{i j q}$ be a binary variable with $w_{i j q}=1$ iff the route leads from location $\mathrm{i}$ to location $\mathrm{j}$ so that $\mathrm{j}$ is reached at position $\mathrm{q}$. Let $t t_{i j}$ denote the traveling time needed to travel from $\mathrm{i}$ to $\mathrm{j}$ including breaks. Using the above definition of sets the following restrictions for the TSPTW can be formulated.

$$
\begin{gathered}
\sum_{i \in I} \sum_{q \in Q(i, j)} w_{i j q}=1 \quad \forall j \in I \\
\sum_{j \in P} w_{0 j 2}=1 \\
\sum_{i \in P} w_{i 0, n+1}=1 \\
\sum_{i \in I(s, q)} w_{i s q}=\sum_{j \in J(s, q+1)} w_{s j, q+1} \forall s \in P, q \in Q(s)
\end{gathered}
$$

Restriction (2) requires that each location is reached exactly once. By (3) and (4) it is required that the depot is left at the beginning of the route and is reached again at position $n+1$. Restriction (5) means: If a location $\mathrm{s}$ is reached at position $\mathrm{q}$ of the route it must be left so that the location which is visited next will be reached at position $\mathrm{q}+1$. If $t_{i}>0$ denotes the arrival time at location $i \in P$, the following restrictions (6) and (7) guarantee that the arrival times at all locations of the route are conform to the traveling times $t t_{i j}$. The restrictions (8) and (9) postulate that the time windows $\left[a_{i}, e_{i}\right]$ for each location i are met. 
Model for the Traveling Salesman Problem including Driving Hours

$$
\begin{gathered}
t_{j} \geq t_{i}+t t_{i j}-M\left(1-\sum_{q \in Q(i, j)} w_{i j q}\right) \forall i \in I, j \in J(i) \backslash\{0\} \\
t_{\text {rueck }} \geq t_{i}+t t_{i 0}-M\left(1-\sum_{q \in Q(i, 0)} w_{i 0 q}\right) \quad \forall i \in P \\
t_{i} \geq a_{i} \forall i \in P \\
t_{i} \leq e_{i} \forall i \in P
\end{gathered}
$$

For modeling the breaks included in the traveling times $t t_{i j}$ the following variables and data are introduced. Let $P 15_{i j q}$ be the integer variable for the number of those 15 minute breaks which are taken between the locations $\mathrm{i}$ and $\mathrm{j}$, while $\mathrm{j}$ is at position $\mathrm{q}$ of the route. Let $P 30_{i j q}$ be the integer variable for the number of second parts of regular breaks with a length of 30 minutes, also between $i$ and $j$ with $j$ at position q. The integer variable $p_{i j q}$ denotes the number of regular breaks separating different driving periods between i and j. Of course, each of these regular breaks can consist of a single break of 45 minutes or two parts with 15 and 30 minutes belonging together to a regular break. Let the variable $T R_{i j}$ denote the duration of a daily rest period taken between the locations $\mathrm{i}$ and $\mathrm{j}$. Then, the traveling times $t t_{i j}$ and $t t_{i 0}$ in (6) and (7) can be calculated by (10) and (11).

$$
\begin{gathered}
t t_{i j}=d_{i j}+\sum_{q \in Q(i, j)}\left(P 15_{i j q} * 0.25+P 30_{i j q} * 0.5\right)+T R_{i j} \\
\forall i \in I, j \in J(i) \backslash\{0\} \\
t_{i 0}=d_{i 0}+\sum_{q \in Q(i, 0)}\left(P 15_{i 0 q} * 0.25+P 30_{i 0 q} * 0.5\right)+T R_{i 0} \quad \forall i \in P
\end{gathered}
$$

The equations (10) and (11) presume that driving between any two customer locations will not require more than one daily rest period. The total driving time necessary to travel from the starting point 0 to the location at the position $\mathrm{q}$ of the route amounts to $G Z_{q}=\sum_{q^{\prime}=1}^{q} \sum_{j \in I} \sum_{i \in I(j, q)} d_{i j} w_{i j q^{\prime}}$. The following constraints (12) to (15) refer to the positioning of the breaks during the route. Constraints (12) and (13) ensure that breaks can only be taken at connections between locations which are part of the route. Constraint (14) requires that there are enough breaks before reaching the location at position 
$\mathrm{q}$, and (15) prevents that breaks are taken in advance in order to use them later on.

$$
\begin{gathered}
p_{i j q}+P 15_{i j q} \leq M * w_{i j q} \forall i, j \in I, q \in Q(i, j) \\
P 30_{i j q} \leq p_{i j q} \forall i, j \in I, q \in Q(i, j) \\
G Z_{q} \leq 4.5\left(\left(\sum_{q^{\prime}=1}^{q} \sum_{j \in I} \sum_{i \in I(j, q)} p_{i j q^{\prime}}\right)+1\right) \quad \forall q \in Q(l), l \in I \\
G Z_{q} \geq 4.5\left(\sum_{q^{\prime}=1}^{q} \sum_{j \in I} \sum_{i \in I(j, q)} p_{i j q^{\prime}}\right) \forall q \in Q(l), l \in I
\end{gathered}
$$

The conditions (16) to (18) arrange the combination of parts of breaks to regular breaks. The constraint (16) causes that up to an arbitrary position q of the route the number of 15-minute-breaks must be greater or equal than the number of regular breaks. Constraint (17) postulates that up to q the number of 30-minute-breaks must be equal to the number of regular breaks and (18) requires that at any position $\mathrm{q}$ at most one 15-minute-break is countable towards input for a regular break. Altogether, the effect is that couples of short breaks of 15 and 30 minutes duration are combined to regular breaks and that a 15minute-break has always to be taken earlier than its corresponding 30-minute-break.

$$
\begin{aligned}
& \sum_{q^{\prime}=1}^{q} \sum_{j \in I} \sum_{i \in I(j, q)} p_{i j q^{\prime}}-\sum_{q^{\prime}=1}^{q} \sum_{j \in I} \sum_{i \in I(j, q)} P 15_{i j q^{\prime}} \leq 0 \quad \forall q \in Q(l), l \in I \\
& \sum_{q^{\prime}=1}^{q} \sum_{j \in I} \sum_{i \in I(j, q)} p_{i j q^{\prime}}-\sum_{q^{\prime}=1}^{q} \sum_{j \in I} \sum_{i \in I(j, q)} P 30_{i j q^{\prime}}=0 \quad \forall q \in Q(l), l \in I \\
& \sum_{q^{\prime}=1}^{q} \sum_{j \in I} \sum_{i \in I(j, q)} p_{i j q^{\prime}}+1 \geq \sum_{q^{\prime}=1}^{q} \sum_{j \in I} \sum_{i \in I(j, q)} P 15_{i j q^{\prime}} \forall q \in Q(l), l \in I
\end{aligned}
$$

The following constraints (19) to (25) formulate the conditions for the positioning and length of the daily rest periods. Let $t p_{i j q}$ be the binary variable with $t p_{i j q}=1$ iff there is a daily rest period between $\mathrm{i}$ and $\mathrm{j}$ at position $\mathrm{q}$. The binary variable $d_{r e d} d_{i j}=1$ iff the daily rest 
period between the locations $\mathrm{i}$ and $\mathrm{j}$ is cut down to a reduced daily rest period. Let $b_{\text {drive }}{ }_{i j q}$ be the binary variable indicating whether on the way from location $\mathrm{i}$ to $\mathrm{j}$ at position $\mathrm{q}$ the daily driving time has exceptionally been extended from 9 to 10 hours.

$$
\begin{gathered}
\text { bdrive } i j q+t p_{i j q} \leq 2 w_{i j q} \forall i, j \in I, q \in Q(i, j) \\
T R_{i j} \geq 11-2 * d r e d_{i j}-M\left(1-\sum_{q \in Q(i, j)} t p_{i j q}\right) \forall i, j \in I \\
\sum_{i \in I} \sum_{j \in I} d r e d_{i j} \leq 3 \\
d r e d_{i j} \leq \sum_{q \in Q(i, j)} w_{i j q} \forall i, j \in I \\
\sum_{q=q^{\prime}}^{q^{\prime \prime}} \sum_{i \in I} \sum_{j \in I} d_{i j} w_{i j q} \leq 9+9\left(\sum_{q=q^{\prime}} \sum_{i \in I} \sum_{j \in I} t p_{i j q}\right)+\sum_{q=q^{\prime}} \sum_{i \in I} \sum_{j \in I} 1 * b d r i v e_{i j q} \\
\forall q^{\prime}, q^{\prime \prime} \in Q(i, j), q^{\prime}<q^{\prime \prime} \\
\sum_{i \in I} \sum_{j \in I} \sum_{q \in Q(i, j)} b d r i v e_{i j q} \leq 2 \\
\sum_{q=q^{\prime}}^{q^{\prime \prime}} b d r i v e_{i j q}-\sum_{q=q^{\prime}}^{q^{\prime \prime}} t p_{i j q} \leq 1 \forall i, j \in I, q^{\prime}, q^{\prime \prime} \in Q(i, j), q^{\prime}<q^{\prime \prime}
\end{gathered}
$$

The condition (19) specifies that daily rest periods and driving time extensions are only possible on used connections $(i, j)$. In (20) it is required that a regular daily rest period must at least last 11 hours and can be reduced by two hours in dependence of the value of $d r e d_{i j}$. Constraint (21) ensures that the possibility to reduce the daily rest period is used at most three times. Restriction (22) states that reductions of daily rest periods are only allowed on used connections. The condition (23) guaranties that for an arbitrary sub-route from any position q' to q" the accumulated driving times are not greater than the total time of the maximal allowed daily driving times that are situated between the positions q' and q". The number of daily driving times situated between them depends on the number of daily rest periods on the considered 
sub-route and on the possible prolongation of single daily driving times by means of bdrive $e_{i j q}$. The inequality (24) states that the extension of the daily driving time can only be applied twice a week. Constraint (25) enables that the daily driving time can be extended up to 10 hours by allowing only one variable bdrive $e_{i j q}$ to equal 1 between two daily rest periods.

The weekly driving time must not exceed 56 hours per single week and 45 hours on average for any two consecutive weeks. Let $W l z_{w-1}$ be the driving time in the previous week, then $\Delta W l z$ denotes the deviation from the average driving time, i.e. $\Delta W l z=45-W l z_{w-1}$. In constraints (26) and (27) the restriction of the weekly driving time is applied to the weekly planning period.

$$
\begin{gathered}
\sum_{i \in I} \sum_{j \in I} \sum_{q \in Q(i, j)} d_{i j} w_{i j q} \leq 56 \\
\sum_{i \in I} \sum_{j \in I} \sum_{q \in Q(i, j)} d_{i j} w_{i j q} \leq 45+\Delta W l z
\end{gathered}
$$

The above objective function (1) and the constraints (2) to (27) yield a complete model for the TSPTW-EU incorporating all rules of the EC Regulation which are statutory for the planning of a single weekly planning period, except the rule that a new daily rest period has to be started at least 24 hours after the end of a daily rest period.

\section{Acknowledgements}

This research was supported by the German Research Foundation (DFG) as part of the Collaborative Research Centre 637 "Autonomous Cooperating Logistics Processes - A Paradigm Shift and its Limitations" (subproject B9).

\section{References}

1. Dethloff J (1994) Verallgemeinterte Tourenplanungsprobleme: Klassifizierung, Modellierung, Lösungsmglichkeiten. Logistik und Verkehr, Göttingen

2. Regulation (EC) No 561/2006 of the European Parliament and of the Council of 15 March 2006, Official Journal of the European Union L $102 / 1,11.4 .2006$

3. Meyer CM, Kopfer H (2008) Restrictions for the operational transportation planning by regulations on drivers' working hours. In: Bortfeldt A, Homberger J, Kopfer H, Pankratz G, Strangmeier R (eds): Intelligent Decision Support. Gabler, Wiesbaden 